\title{
Evolución nutricional y función pulmonar en niños y adolescentes chilenos con fibrosis quística
}

SALESA BARJA ${ }^{1,2}$, TATIANA ESPINOZA ${ }^{4}$, JAIME CERDA ${ }^{3}$, IGNACIO SÁNCHEZ ${ }^{1}$

\section{Growth and pulmonary function in Chilean children and adolescents with cystic fibrosis}

Background: Nutrition influences morbidity and mortality in patients with cystic fibrosis (CF), affecting their lung function. Aim: To characterize the nutritional status of a group of CF patients and to analyze its evolution and relationship to lung function. Patients and Methods: A retrospective cohort of CF children and adolescents attemded in our institution for 15 years, was analyzed. Age and form of presentation, mutation, weight and stature (measured annually at least), microbial colonization and forced expiratory volume in the first second $\left(F E V_{1}\right)$ were registered. Results: We gathered information about 33 patients, 64\% males, diagnosed at 23.8 \pm 45.6 months old (range 1-216), 85\% had a genetic study (10 children had one or more Df508 alleles) and $94 \%$ had pancreatic insufficiency. In their last visit they were $13.0 \pm 5.8$ years old, their body mass index $z$-score (BMIz) was $-0.25 \pm 1.2$ and their FEV was $80.4 \pm 28.6 \%$. According to BMI: $73 \%$ were eutrophic, $18 \%$ undernourished and $9 \%$ were overweight. According to weight/ for height index (WH), the figures for eutrophy, undernutrition and overweight were 70, 6 and 24\%, respectively. Only $12 \%$ had short stature. Those with P. aeruginosa infection had lower BMI. There was a positive correlation between $F E V_{1}$ and BMIz $(+0.46, p=0.02)$, but not with WH. During follow-up, there was a gradual deterioration of weight, height, and BMIz after 10-12 years of age and an overall gradual FEV decrease. Conclusions: The prevalence of malnutrition in these patients with CF is high; undernutrition is higher if defined by BMIz and unlike WH, correlates to lung function. Nutritional deterioration starts before adolescence.

(Rev Med Chile 2011; 139: 977-984).

Key words: Adolescent; Child: Cystic fibrosis; Nutrition.

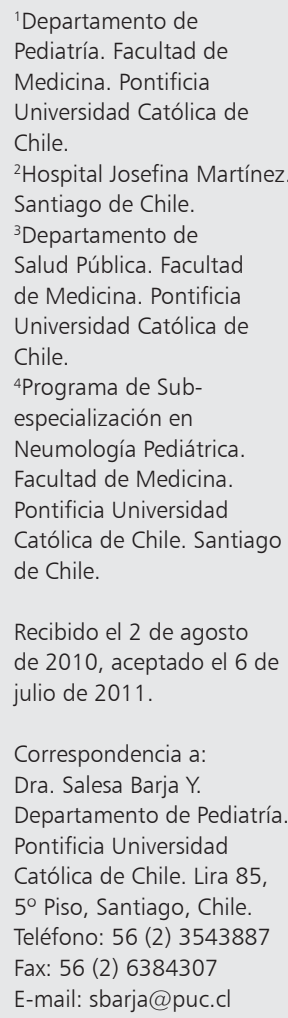

Correspondencia a: Dra. Salesa Barja Y. Departamento de Pediatría. Pontificia Universidad Católica de Chile. Lira 85 $5^{\circ}$ Piso, Santiago, Chile. Teléfono: 56 (2) 3543887 Fax: 56 (2) 6384307 E-mail: sbarja@puc.cl

L a fibrosis quística (FQ) es la enfermedad genética de mayor frecuencia en la edad pediátrica, con un gran desarrollo de la investigación sobre los mecanismos, expresión fenotípica y optimización de su tratamiento, dentro del cual el manejo nutricional es un aspecto fundamental. En estos pacientes diversos factores favorecen la desnutrición, condicionando mayor morbimortalidad, mientras que el estado nutricional normal se asocia a menor morbilidad, mayor sobrevida y mejor calidad de vida ${ }^{1,2}$. Existe una estrecha rela- ción entre el estado nutricional y la evolución de la función pulmonar que, por la naturaleza de esta enfermedad, tiende al compromiso progresivo ${ }^{3}$.

La mejoría de la sobrevida de los pacientes con FQ ha sido posible gracias al diagnóstico precoz, al tratamiento multidisciplinario, a la mejoría de las terapias respiratorias y antiinfecciosas, al igual que del manejo nutricional ${ }^{4}$. En países desarrollados esto último es notorio: en 1997 en Inglaterra se describió que en niños menores a diez años, el peso y talla mejoraron de -1 a $-0,5 \mathrm{DE}$ en 20 años $^{5}$. 
En Estados Unidos de Norteamérica, el índice de masa corporal (IMC) aumentó 5 a 20 puntos percentilares en las diferentes edades entre 1990 y $2006^{3}$. Aunque estos pacientes pueden crecer normalmente, la prevalencia de déficit es aún importante: el año 2002 el 19\% de los menores de 6 años y el 31,5\% de los adolescentes del registro alemán de FQ tenía desnutrición aguda ${ }^{6}$ y en 2006 la Fundación de Fibrosis Quística norteamericana (CFF) reportó $23 \%$ con peso para la edad (P/E) menor al percentil $10^{3}$. La situación en países en vías de desarrollo es menos favorable, con $\mathrm{P} / \mathrm{E}$ $<-2$ DE en $45 \%$ de 124 niños brasileros y en $29,7 \%$ en 128 niños colombianos con $\mathrm{FQ}^{7,8}$.

En Chile, en 2009 había 347 pacientes registrados en el Programa Nacional de FQ, estimándose su sobrevida entre 12 y 18 años de edad. El 45,8\% era mayor a 15 años, con una proporción creciente en servicios médicos de adultos. La prevalencia reportada de desnutrición era 30,2\% $\%^{9}$. Desde el año 2002 existe un Programa Nacional de FQ y en 2007 se publicaron la norma técnica y las guías clínicas para orientar su manejo integral ${ }^{10}$. Sin embargo, no contamos con información específica ni evolutiva en cuanto a su situación nutricional, considerando que existen nuevas recomendaciones internacionales ${ }^{11} \mathrm{y}$ de cara a los grandes desafíos para lograr un mejor cuidado de estos pacientes ${ }^{12}$.

El objetivo de este estudio fue caracterizar el estado y la evolución nutricional de los pacientes con FQ que han sido controlados en nuestra institución y estudiar su relación a la función pulmonar.

\section{Pacientes y Método}

Se realizó un estudio descriptivo de la cohorte de pacientes con FQ controlados en nuestra institución durante los quince años previos a agosto de 2008. Se revisaron las fichas clínicas, completándose la información vía telefónica. Se registró: fecha de nacimiento, sexo, forma de presentación, edad y confirmación diagnóstica, estudio genético, suplementación con enzimas pancreáticas, controles de peso y estatura (al menos anuales), junto a exámenes de laboratorio (esteatocritos ácidos de deposiciones, estudios de función pulmonar y análisis bacteriológicos en expectoración).

Se calificó el estado nutricional mediante el patrón de NCHS-CDC $2000^{13}$, con los índices antropométricos: talla para la edad (T/E), peso para la edad $(\mathrm{P} / \mathrm{E})$, peso para la talla $(\mathrm{P} / \mathrm{T})$ e índice de masa corporal (IMC), expresados como: percentil (p), porcentaje [(valor medido/valor ideal) $\mathrm{x}$ 100] o $\mathrm{z}$-score [(valor medido-mediana) /1 DE]. Se definió obesidad como IMC $\geq$ p95, sobrepeso: p85-94, eutrofia: p10-84 y bajo peso: < p10, éste de acuerdo a recomendaciones para niños con $\mathrm{FQ}^{3,11}$. Se consideró talla baja como zT/E <-2DE.

Se revisó la función pulmonar, representada por el volumen espiratorio forzado del primer segundo $\left(\mathrm{VEF}_{1}\right)$, medido con un espirómetro Schiller SP200. Es normal un $\mathrm{VEF}_{1} \geq 80$; compromiso leve: $\mathrm{VEF}_{1} 65-79$; moderado: VEF 41-64 y severo: $\mathrm{VEF}_{1} \leq 40 \%$. El proyecto fue aprobado por el Comité de Ética Clínica de la Facultad de Medicina de la Pontificia Universidad Católica de Chile.

\section{Análisis estadístico}

Las variables categóricas fueron descritas como frecuencia porcentual y las numéricas como promedio y desviación estándar o mediana y rango. Las variables categóricas fueron comparadas con test de $\chi^{2}$ y las continuas mediante $t$ de Student y ANOVA. Para correlación entre variables numéricas se utilizó el coeficiente de correlación de Pearson. Los cálculos fueron realizados por el software SPSS 15.0, siendo estadísticamente significativo todo valor-p $<0,05$. Para la evolución temporal, se suavizaron las curvas mediante cálculo de promedios móviles.

\section{Resultados}

Se recolectó información de 33 pacientes, $63,6 \%$ de sexo masculino, que consultaron entre enero de 1993 y julio de 2008. En esta fecha, 20 pacientes $(60,6 \%)$ estaban en control regular con neumólogo pediátrico, $4(12,1 \%)$ con neumólogos de adultos y $6(18,2 \%)$ en otros centros. Tres pacientes $(9,1 \%)$ fallecieron a los 14,19 y 23 años de edad.

La edad media de diagnóstico de FQ fue de 23,8 \pm 45,6 meses (mediana: 7,0 meses, rango 1 a 216$)$. Ocho pacientes $(24,2 \%)$ en período neonatal: 5 por íleo meconial (62\%), 1 por prolapso rectal $(12,5 \%), 1$ por neumonía $(12,5 \%)$ y 1 con el antecedente de hermano con FQ (12,5\%). Catorce niños $(42,4 \%)$ fueron diagnosticados entre el mes y el año de vida y $11(33,3 \%)$ posteriormente, por 
Tabla 1. Características clínicas, estado nutricional y función pulmonar (VEF $)$ de 33 niños y adolescentes con fibrosis quística

\begin{tabular}{|lccc|}
\hline & Promedio (DE) & Mediana & Rango \\
\hline Edad (años) & $13,0 \pm 5,8$ & 13,0 & 2,$0 ; 20,0$ \\
\hline Peso $(\mathrm{kg})$ & $39,6 \pm 15,8$ & 42,0 & 11,$7 ; 65,0$ \\
Talla $(\mathrm{cm})$ & $141,9 \pm 26,0$ & 150,0 & 80,$0 ; 174,0$ \\
IMC $\left(\mathrm{kg} / \mathrm{m}^{2}\right)$ & $18,7 \pm 3,0$ & 18,7 & 15,$0 ; 28,8$ \\
z P/E & $-0,71 \pm 1,29$ & $-0,58$ & $-3,54 ;+1,37$ \\
z T/E & $-0,88 \pm 1,01$ & $-0,95$ & $-3,13 ;+2,14$ \\
zIMC & $-0,25 \pm 1,23$ & $-0,25$ & $-3,54 ;+1,98$ \\
VEF,$(\%) *$ & $80,4 \pm 28,6$ & 80 & $31 ; 135$ \\
\hline
\end{tabular}

$\mathrm{VEF}_{1}$ : Volumen espiratorio forzado del primer segundo. *Disponible en 25 pacientes.

infecciones respiratorias recurrentes $(45 \%)$, desnutrición (30\%) u otros (25\%). En todos se contó con al menos dos estudios anormales de test del sudor (medido por conductividad, ratificado por determinación cuantitativa de electrolitos), cuyo valor fue de 92,6 $\pm 13,7 \mathrm{meq} \mathrm{Cl} / \mathrm{dL}$ (mediana: $\mathrm{Cl}$ $90 \mathrm{mEq} / \mathrm{dL}$, rango: $67-117)$. Veintiocho $(84,8 \%)$ tenía estudio genético, $10(35,7 \%)$ con uno o más alelos Df508.

La Tabla 1 muestra la edad, antropometría y $\mathrm{VEF}_{1}$ de los pacientes. Estado nutricional: según su IMC, 24 pacientes estaban eutróficos $(72,7 \%), 3$ tenían sobrepeso $(9,1 \%)$ y 6 desnutrición $(18,2 \%)$. Utilizando el IPT, 23 eran eutróficos $(69,7 \%), 8$ tenían sobrepeso $(24,2 \%)$ y 2 desnutrición $(6,1 \%)$ (Tabla 2). Cuatro pacientes tenían talla baja (T/E $<-2 \mathrm{DE}), 12$ talla en rango normal-bajo (T/E entre -1 y $-2 \mathrm{DE})$ y 17 normal (T/E -1 a +2DE). Según $\mathrm{P} / \mathrm{E}, 14$ (42,4\%) estaba bajo -1DE y 4 (12,1\%) bajo

\section{Tabla 2. Diagnóstico nutricional de acuerdo a IMC e IPT en 33 niños y adolescentes con fibrosis quística}

\begin{tabular}{|lcr|}
\hline $\begin{array}{l}\text { Diagnóstico } \\
\text { nutricional }\end{array}$ & IMC & \multicolumn{1}{c|}{ IPT } \\
\hline Eutrofia & $24(72,7 \%)$ & $23(69,7 \%)$ \\
Sobrepeso & $3(9,1 \%)$ & $8(24,2 \%)$ \\
Desnutrición & $6(18,2 \%)$ & $2(6,1 \%)$ \\
\hline
\end{tabular}

IMC: Índice de Masa Corporal. IPT: Índice Peso/Talla (\%). Estado nutricional: Eutrofia: IMC p10-84 o IPT 90-109\%, Sobrepeso: IMC $\geq$ p85 o IPT $\geq 110 \%$, Desnutrición: IMC < p10 o IPT $<90 \%$.
-2DE. Considerando la distribución según IMC, el $45 \%$ de los pacientes se encontraba bajo p50.

El $94 \%$ presentaba insuficiencia pancreática, habiendo comenzado tempranamente la suplementación enzimática. Los esteatocritos ácidos de deposiciones fueron recuperados en $80 \%$ de los pacientes y estaban en rango normal en su último control.

En cuanto a función pulmonar, se contó con espirometrías en 25 pacientes, todos mayores a 6 años de edad ( 16 hombres y 9 mujeres, con edad $14,7 \pm 4,7$ años). En su último control el $\mathrm{VEF}_{1}$ fue de $80,4 \pm 28,6 \%$ (mediana $77 \%$, rango 31 a 135 ). El 48,0\% (12/25) estaba en rango normal, 28,0\% (7/25) presentaba compromiso leve, $12,0 \%(3 / 25)$ moderado y $12,0 \%(3 / 25)$ severo. Se observó una asociación directa entre función pulmonar $\left(\mathrm{VEF}_{1}\right)$ y estado nutricional valorado con zIMC $(r=+0,459 ; p=0,02)$, pero no con IPT $(r=+0,151$; $\mathrm{p}=0,47)$, (Figuras 1 y 2 ).

El zIMC fue menor en los pacientes colonizados por $P$. aeruginosa: $-1,04 \pm 1,52$ vs $+0,28 \pm 1,03$ en los no colonizados $(\mathrm{p}=0,02)$, sin encontrarse diferencias significativas para zIMC según edad de diagnóstico $(-0,12 \pm 0,92$ neonatal, $-0,25 \pm 1,32$ entre 1 y12 meses y $-0,35 \pm 1,41$ en mayores de 1 año, $p>0,05)$. Tampoco hubo diferencia según sexo $(-0,47 \pm 1,35$ en hombres vs $+0,13 \pm 0,93$ en mujeres, $\mathrm{p}=0,18)$ o mutación $(-0,25 \pm 1,05$ con algún alelo DF508 vs $-0,01 \pm 1,21$ el resto, $\mathrm{p}=0,60$ ).

El tiempo de seguimiento fue de $8,8 \pm 4,2$ años (mediana: 8 años, rango 2-15). En cuanto a la evolución nutricional, en la Figura 3 se observa la tendencia de los promedios móviles de zP/E y 


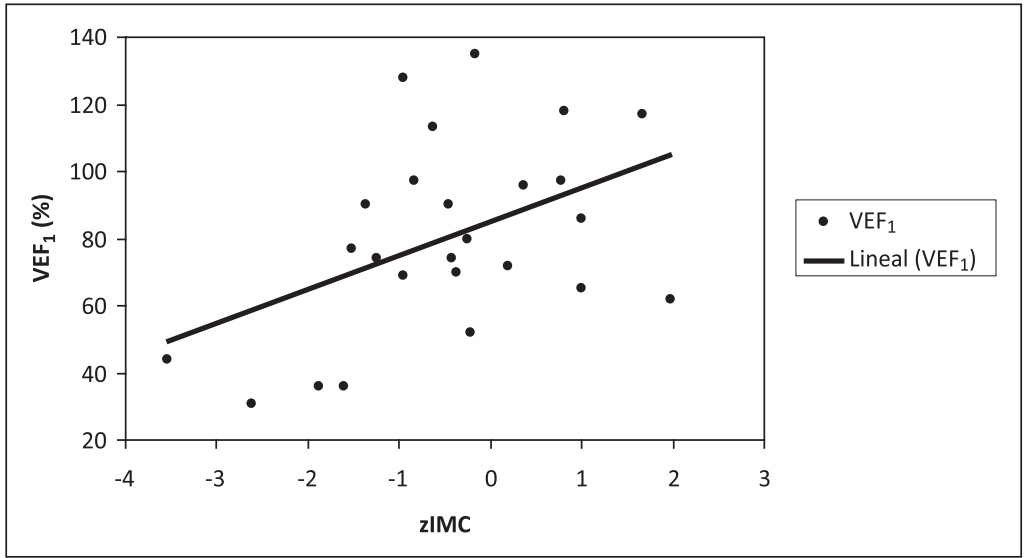

Figura 1. Correlación entre la función pulmonar $\left(\mathrm{VEF}_{1}\right)$ y el estado nutricional $(z \mid M C)$ en 25 niños y adolescentes con fibrosis quística. $V F_{1}$ : Volumen espiratorio forzado del primer segundo; zIMC: Score z para índice de masa ponderal = [(IMC medido-IMC mediana) /1 DE]. Coeficiente de correlación de Pearson: $r=+0,459(p=0,02)$.

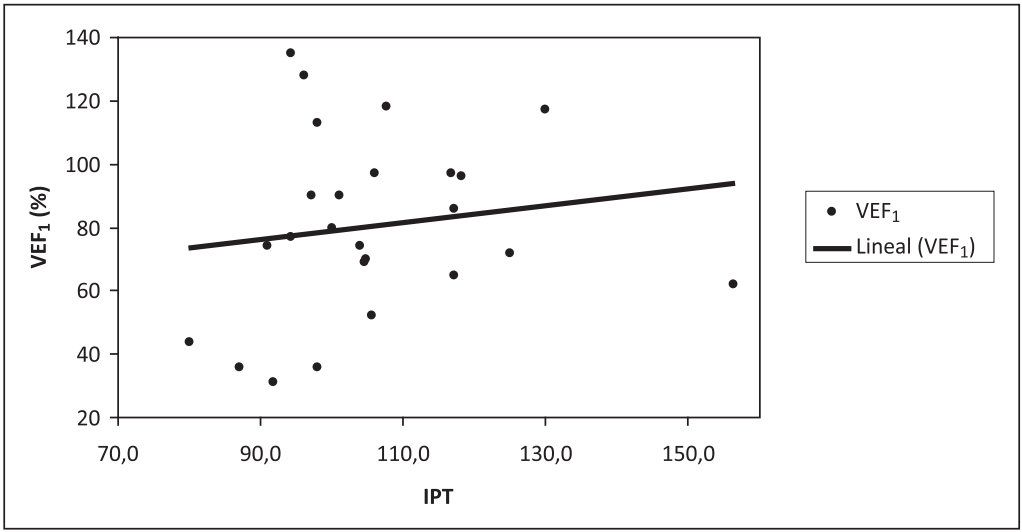

Figura 2. Correlación entre la función pulmonar $\left(\mathrm{VEF}_{1}\right)$ y el estado nutricional (IPT) en 25 niños y adolescentes con fibrosis quística. $V F_{1}$ : Volumen espiratorio forzado del primer segundo. IPT: Índice de peso para la talla = [(Peso medido/Peso ideal para la talla) $x$ 100]. Coeficiente de correlación de Pearson: $r=+0,151(p=0,47)$.

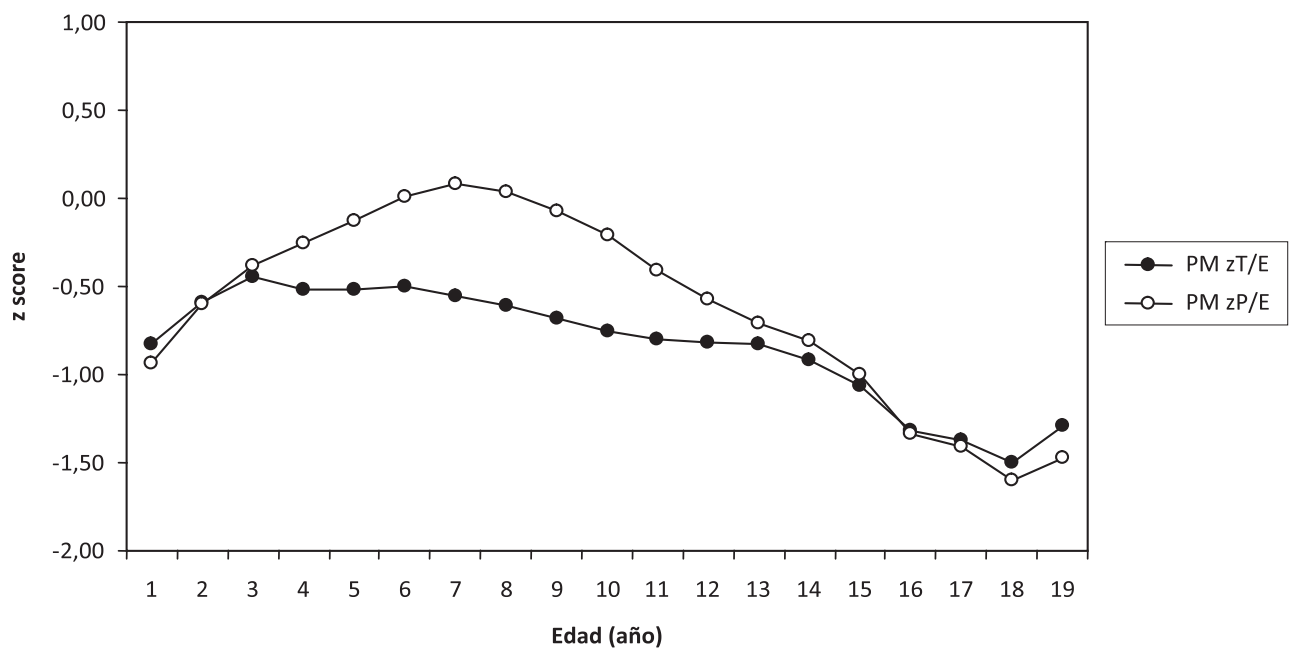

Figura 3. Evolución de los promedios móviles de z P/E y z T/E en 33 niños y adolescentes con fibrosis quística, de acuerdo a la edad. zP/E: z score para Índice Peso/Edad= [(Peso medido- Peso mediana) /1 DE]. zT/E: z score para Índice Talla/Edad= [(Talla medida- Talla mediana) /1 DE]. 

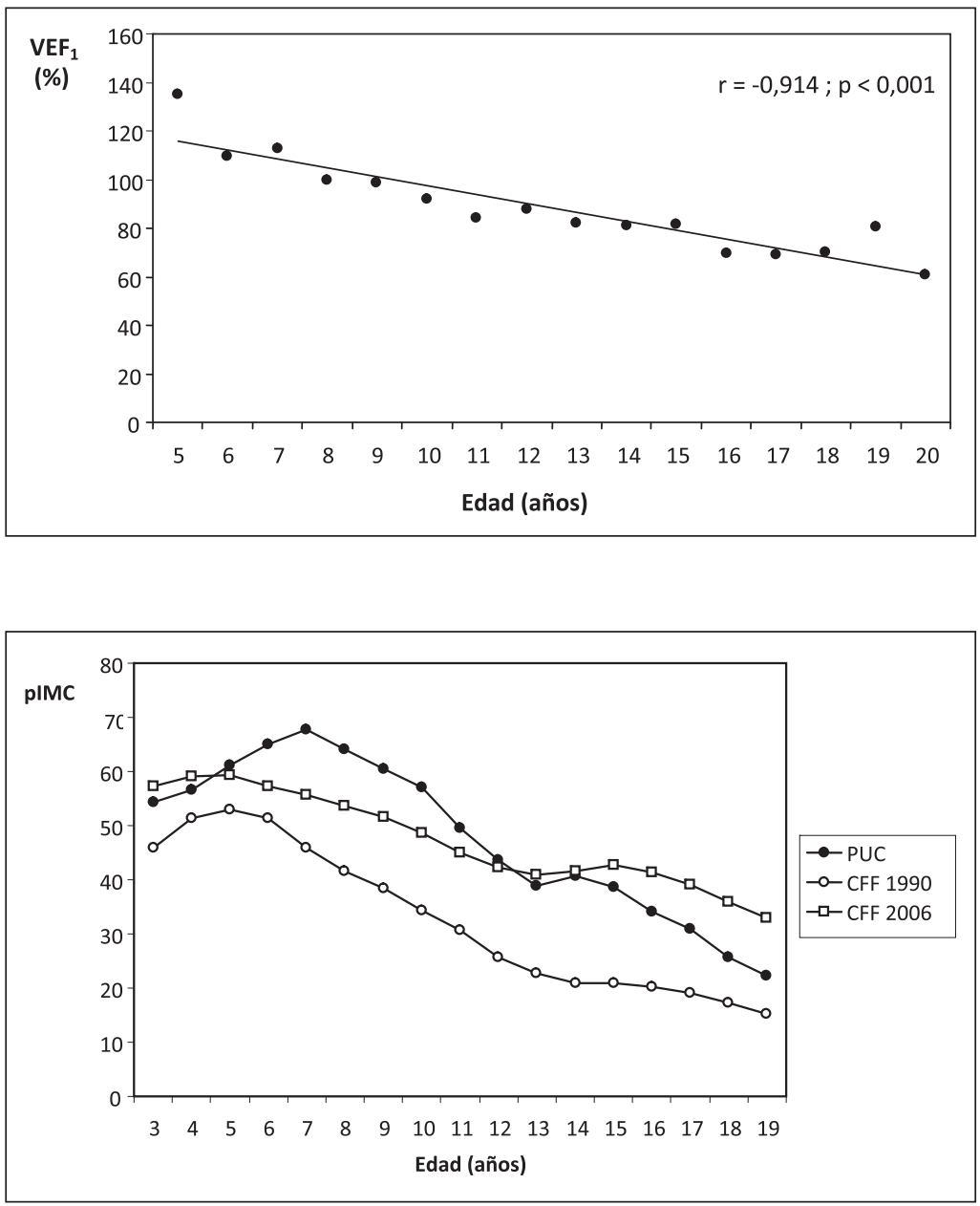

Figura 4. Evolución de la función pulmonar $\left(V_{E} F_{1}\right)$ en 25 pacientes con $F Q$, de acuerdo a la edad. $V E F_{1}$ : Volumen espiratorio forzado del primer segundo. Coeficiente de correlación de Pearson: $r=-0,946(p=<0,01)$.

Figura 5. Evolución del promedio móvil de p IMC en 33 niños con Fibrosis Quística, comparado a las reportadas por la Fundación de fibrosis quística Norteamericana en 1990 y 2006. IMC: Índice de masa corporal; p IMC: Percentil de IMC. CFF: Fundación de fibrosis quística norteamericana ${ }^{3}$.
zT/E en relación a la edad. Con respecto al peso, se distingue una primera fase de aumento del zP/E entre las edades 1-7 años y una reducción entre los 8-19 años. En cuanto a la talla, se observa un patrón descendente del zT/E entre los 3 a 19 años (el cálculo de promedios móviles anula los datos de las edades $<1$ y 20 años).

En la Figura 4 se observa la tendencia temporal del $\mathrm{VEF}_{1}$, la curva tiene una reducción global entre los 5 y 19 años de edad, con correlación de $-0,914(p<0,001)$. Finalmente, en la Figura 5 se observa la evolución del IMC (en percentiles), con un incremento hasta los 6 años, seguido por un lento descenso hasta los 11 años y una disminución pronunciada posterior. Aparecen también como referencia las curvas de la CFF de 1990 y $2006^{3}$, ubicándose la nuestra en posición intermedia, con tendencia a mayor cercanía a la última, pero con mayor deterioro en la adolescencia.

Los pacientes fallecidos eran de sexo masculino y tenían en su último control, las siguientes características: Paciente 1: 11 años, zIMC: $-0,84$, $\mathrm{VEF}_{1}$ : (no realizable por enfermedad neurológica de base); paciente 2: 14 años, zIMC: $-1,61, \mathrm{VEF}_{1}$ : $36 \%$ y paciente $3: 17$ años, zIMC: $-3,54, \mathrm{VEF}_{1}: 44 \%$.

\section{Discusión}

El presente trabajo describe el estado nutricional, la evolución de éste y de la función pulmonar en un grupo de 33 niños portadores de FQ. Es una cohorte no concurrente de tamaño muestral limitado, pero la primera en publicarse en nuestro país. 
La prevalencia de $18,2 \%$ de desnutrición encontrada utilizando IMC es comparable a series extranjeras, aunque en éstas se han utilizado diferentes índices antropométricos, puntos de corte y patrones de referencia, haciendo necesario un lenguaje común. En FQ tradicionalmente se ha usado el P/E para describir prevalencia de desnutrición ${ }^{7,8,16}$ e IPT para decidir conductas de apoyo nutricional ${ }^{17-19}$. Dada su relevancia, en 2005 la CFF encargó a un grupo de expertos analizar los criterios diagnósticos y objetivos nutricionales, a la luz de la evidencia científica disponible ${ }^{11}$. Este comité recomendó utilizar el IMC, por su mayor sensibilidad para detectar desnutrición y asociación más estrecha a la función pulmonar que el IPT ${ }^{20}$. Este último sobre-estima sobrepeso y sub-diagnostica déficit en niños con talla baja, hecho frecuente en estos pacientes y evidenciado en nuestro análisis ${ }^{21,22}$. Al contrario, el $\mathrm{P} / \mathrm{E}$ es más sensible para detectar desnutrición, pero la sobreestima en niños con talla baja de otro origen (por ejemplo, familiar).

Encontramos $12 \%$ con $\mathrm{P} / \mathrm{E}<-2 \mathrm{DE}$, menor que otras series latinoamericanas ${ }^{7,8}$, estando en nuestro estudio todos los desnutridos en la adolescencia ${ }^{18}$. Algunos factores a considerar para explicar la mejor situación nutricional son: el predominio leve del sexo masculino, que suele presentar menor déficit nutricional que el femenino ${ }^{6,14}$ y la menor edad de diagnóstico que otras reportadas ${ }^{7,8,15}$. Sin embargo, si bien esta comparación es favorable, es necesario destacar la baja proporción de niños diagnosticados antes del mes de vida (24,2\%), aspecto posible de mejorar con mayor índice de sospecha precoz. También influye la relativa preservación de la función pulmonar, normal en $48 \%$ de los pacientes capaces de realizar la espirometría. Limitaciones propias de la muestra, como el amplio rango etáreo y la heterogeneidad del seguimiento influyen también en la interpretación del estado nutricional de la misma.

Aunque los puntos de corte de estado nutricional asociados a función pulmonar aceptable están aún en discusión ${ }^{22,23}$, la CFF recomendó como meta el mantener un IMC $\geq$ p50 en niños y adolescentes. En nuestro estudio, $45 \%$ está bajo este límite y aunque en $200657 \%$ de los pacientes de la CFF también lo estaba, aquella es una muestra de gran tamaño y mayor sobrevida, demostrando que es un objetivo ambicioso, pero posible ${ }^{17,22}$. Dicha recomendación se basa en que sobre p50 de IMC, el $\mathrm{VEF}_{1}$ presenta un plateau superior a $80 \%$ y bajo p50 se deteriora, con mayor pendiente bajo $\mathrm{p} 10^{3}$. Para adelantarse, se recomienda apoyo nutricional precoz y activo ${ }^{10,11,24}$, siendo importante explicar el déficit y acordar con el paciente y su familia los plazos de respuesta, ya que en etapas avanzadas de la enfermedad se suman otros factores difíciles de revertir, los cuales agravan el deterioro pulmonar $^{25-27}$. En adultos, se recomienda mantener un IMC mayor a 22 en mujeres y a 23 en hombres, puntos de corte asociados a un $\mathrm{VEF}_{1} \geq 60 \%$ del predicho ${ }^{11}$.

La correlación directa entre $\mathrm{VEF}_{1}$ e IMC fue significativa, a pesar del tamaño muestral, siendo comparable a la reportada en niños australianos con enfermedad leve ${ }^{28}$. Aún no está claro el mecanismo de esta interacción que probablemente depende de la preservación de masa magra, con mejor contractibilidad de los músculos respiratorios $^{24,29}$. Algunos estudios demuestran que la intervención nutricional puede asociarse a mejoría del $\mathrm{VEF}_{1}^{30,31}$ o al menos a desaceleración de su deterioro $^{26}$.

Es necesario destacar que tres pacientes tenían exceso de peso, todos menores de 11 años de edad, período de estabilidad nutricional. El sobrepeso puede influir negativamente en la función pulmonar, al igual que favorecer complicaciones asociadas a la FQ, tales como diabetes, dislipidemias y esteatosis hepática. Si bien se ha planteado que por el mayor riesgo de desnutrición posterior el sobrepeso se corregirá, ello requiere ser demostrado con estudios de seguimiento, siendo recomendable el evitar la obesidad.

El retraso de talla es frecuente y multifactorial en los pacientes con $\mathrm{FQ}$, en general asociado a diagnóstico tardío o desnutrición; Reis reportó $26,5 \%$ de talla baja y Vásquez $36 \%{ }^{7,8}$, ambos superiores al $12 \%$ en nuestro estudio. Influyen en ello las características de cada muestra, siendo una limitación común el no corregir por la carga genética parental. Por otra parte, se ha considerado necesario re-definir los puntos de corte para diagnosticar retraso de talla en estos pacientes, dando mayor énfasis a la velocidad de crecimiento ${ }^{22}$.

En cuanto a colonización por $P$. aeruginosa, el tamaño muestral limita su análisis, pero el menor IMC en los portadores coincide con la literatura. Dicha asociación se explica porque la colonización aumenta en la pre-adolescencia, la cual conlleva per se altos requerimientos energéticos, acentua- 
dos por la mayor frecuencia de exacerbaciones que producen además anorexia, disminuyendo la ingesta de alimentos y empeorando la desnutrición ${ }^{31,32}$.

La evolución del estado nutricional de estos 33 pacientes se sitúa en posición intermedia en relación a los reportes de 1990 y 2006 de la CFF. Aunque nuestra curva tiene mayor variabilidad, por su menor tamaño muestral, el P/E y T/E tienen curso similar, aunque la talla se compromete antes. El IMC se mantiene sobre p50 hasta los 11 años, con mayor deterioro posterior. Observamos un mayor compromiso de la función pulmonar que en la CFF, considerando que en ésta la mejoría histórica del $\mathrm{VEF}_{1}$ ha sido inferior que la de los índices antropométricos, planteándose que las infecciones y su manejo tienen un impacto considerable ${ }^{33}$. Así, el estado nutricional óptimo es un factor protector, pero el apoyo nutricional puede ser inefectivo en etapas avanzadas ${ }^{24}$.

En resumen, el presente estudio en pacientes pediátricos con $\mathrm{FQ}$ muestra una prevalencia importante de desnutrición que varía según el índice antropométrico utilizado y que se asocia a deterioro de la función pulmonar. Se confirma que el IMC tiene correlación directa con el $\mathrm{VEF}_{1}$. En el tiempo, destaca una fase inicial de recuperación, una intermedia de estabilidad nutricional entre los 3 y 10 años de edad y un posterior deterioro. Dada la mayor sobrevida de los pacientes con FQ, es mandatorio mejorar su evolución nutricional, en especial en la adolescencia, etapa crítica para enfrentar el paso a la edad adulta en las mejores condiciones $^{34}$. Ello será posible mediante la detección precoz del déficit y el uso de mejores índices y puntos de corte, para optimizar la nutrición y contribuir a preservar la función pulmonar. Se requiere además de estudios multicéntricos que muestren el espectro de la realidad chilena, necesarios para mejorar este importante aspecto de la enfermedad, que incide directamente en su morbimortalidad.

\section{Referencias}

1. Sharma R, Florea VG, Bolger AP, Doehner W, Florea $\mathrm{ND}$, Coats AJ, et al. Wasting as an independent predictor of mortality in patients with cystic fibrosis. Thorax 2001; 56: 746-50.

2. Corey M, McLaughlin FJ, Williams M, Levison H.
A comparison of survival, growth, and pulmonary function in patients with cystic fibrosis in Boston and Toronto. J Clin Epidemiol 1988; 41: 583-91.

3. Cystic Fibrosis Foundation, Patient Registry: 2002 and 2006 Annual Report, Bethesda, Maryland.

4. Bell SC, Shepherd RW. Optimizing nutrition in cystic fibrosis. J Cyst Fibros 2002; 1: 47-50.

5. Morison S, Dodge JA, Cole TJ, Lewis PA, Coles EC, Geddes D, et al. The UK Cystic Fibrosis Survey Management Committee. Height and weight in cystic fibrosis: a cross-sectional study. Arch Dis Child 1997; 77: 497-500.

6. Steinkamp G, Wiedemann B, on behalf of the German CFQA Group. Relationship between nutritional status and lung function in cystic fibrosis: cross sectional and longitudinal analyses from the German CF quality assurance (CFQA) project. Thorax 2002; 57: 596-601.

7. Reis F, Oliveira M, Penna F, Oliveira M, Oliveira E, Monteiro A, et al. Quadro clínico e nutricional de pacientes com Fibrose Cística: 20 anos de seguimento no HCUFMG. Rev Med Brasil 2000; 46: 325-30.

8. Vásquez C, Aristizával R, Daza W. Fibrosis Quística en Colombia. Rev Chil Neumol Ped 2010; 5: 44-50.

9. Astudillo P. Fibrosis Quística en Chile. Rev Chil Neumol Ped 2010; 5: 42-3.

10. Barja S, Rebollo MJ, Reyes M, González M, Chávez E, Jofré P. Comisión Técnica, Programa Nacional de Fibrosis Quística: Manual técnico de diagnóstico y tratamiento de la Fibrosis Quística 2007: Aspectos nutricionales y gastroenterológicos. Disponible en: www.fibrosisquisticachile.cl

11. Stallings, VA, Stark LJ, Robinson KA, Feranchack A, Quinton H. Evidence-based practice. Recommendations for nutrition-related management of children and adults with Cystic Fibrosis and pancreatic insufficiency: Results of a systematic review: clinical practice guidelines on growth and nutrition subcommittee; ad hoc working group. J Am Diet Assoc 2008; 108: 832-9.

12. Gutiérrez H, Sánchez I, Schidlow DV. Cystic fibrosis care in Chile. Curr Opin Pulm Med 2009; 15: 632-7.

13. Kuczmarski RJ, Ogden C, Grummer-Strawn ML, Flegal KM, Guo SS, Wei R, et al. CDC growth Charts: United States. Hyattsville MD: US Department of Health and Human Services, 2000. NCHS Advance Data Report no 314. Accedido el 11 de abril 2011. Disponible en: http:// www.cdc.gov/nchs/data/ad/ad314.pdf

14. Stallings VA, Tomezsko JL, Schalla JI, Mascarenhas MR, Stettler N, Scanlin TF, et al. Adolescent development and energy expenditure in females with cystic fibrosis. Clin Nutr 2005; 24, 737-45.

15. Macri CN, De Gentile AS, Manterola A, Tomezzoli S, Reis FC, Largo García I, et al. Epidemiology of cystic 
fibrosis in Latin America: preliminary communication. Pediatr Pulmonol 1991; 10: 249-53.

16. Ramsey BW, Farrell PM, Pencharz P. Nutritional assessment and management in cystic fibrosis: a consensus report. The Consensus Committee. Am J Clin Nutr 1992; 55: 108-16.

17. Sinaasappel M, Stern M, Littlewood J, Wolfe S, Steinkamp G, Heijerman HG, et al. Nutrition in patients with cystic fibrosis: a European Consensus. J Cyst Fibros 2002; 1: 51-75.

18. Borowitz D, Baker RD, Stallings V. Consensus report on nutrition for pediatric patients with cystic fibrosis. J Pediatr Gastroenterol Nutr 2002; 35: 246-59.

19. Sánchez I, Pérez MA, Boza ML, Lezana V, Vila A, Repetto G, et al. Consenso nacional de Fibrosis Quística. Rev Chil Pediatr 2001; 72: 356-80.

20. Wiedemann B, Paul KD, Stern M, Wagner TO, Hirche TO; German CFQA Group. Evaluation of body mass index percentiles for assessment of malnutrition in children with cystic fibrosis. Eur J Clin Nutr 2007; 61: 759-68.

21. Zhang Z, Lai HJ. Comparison of the use of body mass index percentiles and percentage of ideal body weight to screen for malnutrition in children with cystic fibrosis. Am J Clin Nutr 2004; 80: 982-91.

22. Lai HJ. Classification of nutritional status in cystic fibrosis. Curr Opin Pulm Med 2006; 12: 422-7.

23. Lai HJ, Shoff SM. Classification of malnutrition in cystic fibrosis: implications for evaluating and benchmarking clinical practice performance. Am J Clin Nutr 2008; 88: 161-6.

24. Milla CE. Nutrition and lung disease in cystic fibrosis. Clin Chest Med 2007; 28: 319-30.

25. Barja S, Rebollo MJ. Manejo nutricional de niños y adolescentes con Fibrosis Quística. Rev Chil Pediatr 2009; 80: $274-84$.

26. Efrati O, Mei-Zahav M, Rivlin J, Kerem E, Blau H, Barak
A, et al. Long Term Nutritional Rehabilitation by Gastrostomy in Israeli Patients with Cystic Fibrosis: Clinical Outcome in Advanced Pulmonary Disease. J Pediatr Gastroenterol Nutr 2006; 42: 222-8.

27. Conway SP, Morton A, Wolfe S. Enteral tube feeding for cystic fibrosis. Cochrane Database of Systematic Reviews 2008, Issue2. Art.No.CD001198.DOI: 10.1002/14651858. CD001198.pub2.

28. Pedreira CC, Robert RG, Dalton V, Oliver MR, Carlin JB, Robinson P, et al. Association of body composition and lung function in children with cystic fibrosis. Pediatr Pulmonol 2005; 39: 276-80.

29. Hart N, Tounian P, Clément A, Boulé M, Polkey MI, Lofaso $F$, et al. Nutritional status is an important predictor of diaphragm strength in young patients with cystic fibrosis. Am J Clin Nutr 2004; 80: 1201-6.

30. Goldbart AD, Cohen AD, Weitzman D, Tal A. Effects of rehabilitation winter camps at the Dead Sea on European cystic fibrosis patients. Isr Med Assoc J 2007; 9: 806-9.

31. McPhail GL, Acton JD, Fenchel MC, Amin RS, Seid M. Improvements in lung function outcomes in children with cystic fibrosis are associated with better nutrition, fewer chronic pseudomonas aeruginosa infections, and dornase alfa use. J Pediatr 2008; 153: 752-7.

32. Steinkamp G, Drommer A, von der Hardt H. Resting energy expenditure before and after treatment for Pseudomonas Aeruginosa infection in patients with cystic fibrosis. Am J Clin Nutr 1993; 57: 685-9.

33. Zemanick ET, Harris JK, Conway S, Konstan MW, Marshall B, Quittner AL, et al. Measuring and improving respiratory outcomes in cystic fibrosis lung disease: Opportunities and challenges to therapy. J Cyst Fibros 2010; 9: 1-16.

34. Vega-Briceño L, Guiraldes E, Sánchez I. Fibrosis Quística: Enfrentando la transición desde el pediatra hacia el internista. Rev Med Chile 2006; 134: 365-71. 